\title{
Signatures of spin-related phases in transport through regular polygons
}

\author{
Dario Bercioux \\ Institut für Theoretische Physik, Universität Regensburg, D-93040, Germany \\ Coherentia-INFM $\&$ Dipartimento di Scienze Fisiche, \\ Università degli Studi "Federico II", I-80126 Napoli, Italy \\ Diego Frustaglia and Michele Governale \\ NEST-INFM \& Scuola Normale Superiore, I-56126 Pisa, Italy
}

(Dated: July 1, 2021)

\begin{abstract}
We address the subject of transport in one-dimensional ballistic polygon loops subject to Rashba spin-orbit coupling. We identify the role played by the polygon vertices in the accumulation of spinrelated phases by studying interference effects as a function of the spin-orbit coupling strength. We find that the vertices act as strong spin-scattering centers that hinder the developing of AharovovCasher and Berry phases. In particular, we show that the oscillation frequency of interference pattern can be doubled by modifying the shape of the loop from a square to a circle.
\end{abstract}

PACS numbers: 03.65.Vf,71.70.Ej,73.23.-b

Many efforts have been done towards the study of spin effects at the mesoscopic scale since the original DattaDas proposal ${ }^{1}$ for a spin-field effect transistor. This is based on the control of the Rashba spin-orbit (SO) coupling $^{2}$ in low-dimensional electron gas subject to asymmetric quantum confinement. As a consequence, a large variety of alternative setups relying on similar principles has been presented. Systems of particular interest are the spin interferometers 3.4 and their extension to quantum networks ${ }^{5}$ which do not require the injection of spinpolarized carriers as a working principle. Instead, they work by only tuning the Aharonov-Casher (AC) phase ${ }^{6}$ acquired by spin carriers in the presence of SO coupling. Spin interferometers are interesting not only in view of possible spintronics 7 applications but also from a fundamental perspective regarding the study of spin dynamics and related quantum phases. For instance, conducting rings have been proposed ${ }^{8}$ as paradigmatic systems for the identification of geometrical or Berry phases $\frac{9}{}$ with relative experimental success ${ }^{10.11}$. Berry phases arise when the spins suffer an adiabatic evolution during transport, i.e., when they adiabatically follow the local direction of the effective magnetic field during transport (see e.g. Ref. 4). Among the different factors that can affect the spin evolution (and consequently the eventual presence of Berry phases) it stands out disorder, a subject extensively discussed in the literature ${ }^{12}$. Surprisingly, less interest has been put on other relevant geometrical aspects. Only recently Yang et $a l^{11}$ proposed alternative setups optimizing the contacts to the leads in order to avoid eventual non-adiabatic spin flipping, and van Veenhuizen et al. ${ }^{13}$ discussed single-probe spininterference features in closed polygon-structures.

In this Brief Report we discuss the two-contact transport properties of regular polygons subject to Rashba SO coupling. We approach the subject by means of the Landauer-Büttiker ${ }^{14}$ formulation, identifying the linear conductance with quantum transmission. We calculate the linear conductance of several polygons made of one-

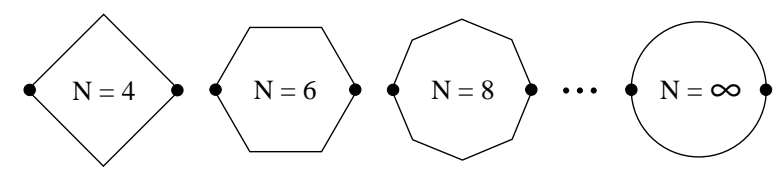

FIG. 1: Series of regular-polygon conductors of constant perimeter. Vertices are connected by single-channel ballistic quantum wires with SO coupling. In the limit of infinite number of vertices the series converges to a single-channel circular conductor. The full dots represent the point where input and output leads are attached.

dimensional (1D) ballistic (disorder free) wires as a function of the SO strength. As a result we obtain a series of interference patterns reproducing the characteristics of the spin-related phase accumulation. This permit us to identify the role played by non-adiabatic spin-scattering taking place at the vertices of the polygons.

We consider electron transport through regular polygons with an even number of vertices $N$ as those shown in Fig. 1] The vertices are connected by single-channel ballistic quantum wires subject Rashba SO coupling. The polygons are symmetrically coupled to two 1D leads free of SO coupling at opposite vertices. This model neglects the subband hybridization due to the Rashba effect ${ }^{15}$. (See Ref. 5 for a related model). The Hamiltonian for a single-channel wire along a generic direction $\hat{\gamma}$ in the $x-y$ plane reads

$$
\mathcal{H}=\frac{p_{\gamma}^{2}}{2 m}-\frac{\hbar k_{\mathrm{SO}}}{m} p_{\gamma}(\hat{z} \times \hat{\gamma}) \cdot \vec{\sigma}
$$

where $k_{\mathrm{SO}}$ is the SO coupling strength, and $\vec{\sigma}$ is the vector of the Pauli matrices. The second term in Eq. (11) can be viewed as a Zeeman splitting due to a momentumdependent, in-plane, effective magnetic field. The SO coupling strength $k_{\mathrm{SO}}$ is related to the spin precession length $L_{\mathrm{SO}}$ by $L_{\mathrm{SO}}=\pi / k_{\mathrm{SO}}$. For InAs quantum wells the spin-precession length ranges from 0.2 to $1 \mu \mathrm{m}^{16}$. 


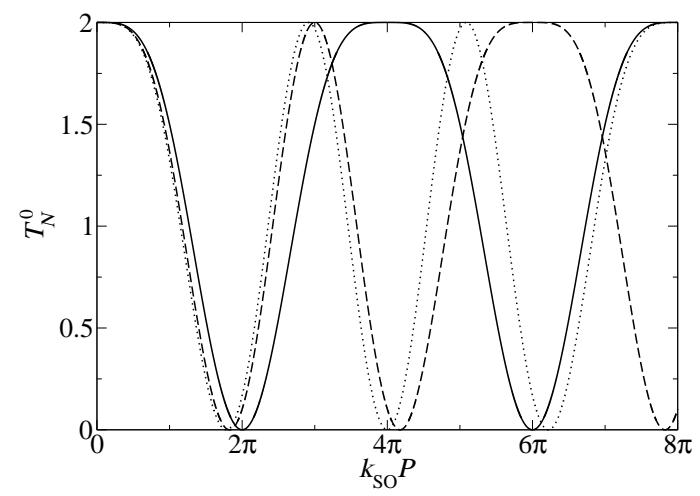

FIG. 2: Lowest order transmission as function of the dimensionless SO coupling strength for polygons with $N=4$ (solid line), $N=6$ (dashed line) and $N=8$ (dotted line).

The spin dynamics along one side of a polygon can be described by a spin-rotation operator (SRO) that accounts for spin precession around the effective in-plane magnetic field due to the SO coupling ${ }^{5}$ :

$$
\mathcal{R}_{q, p}\left(k_{\mathrm{SO}}\right)=\exp \left\{-i \vec{\sigma} \cdot\left(\hat{z} \times \hat{\gamma}_{q, p}\right) k_{\mathrm{SO}} l_{q, p}\right\},
$$

where $\hat{\gamma}_{q, p}$ and $l_{q, p}$ are the orientation and length of the bond connecting the vertices $p$ and $q$, respectively. The vertices are numbered clockwise from 1 to $N(N$ even). The incoming (outgoing) lead is coupled to vertex $1(N / 2+1)$. For regular polygons it is $l_{q, p}=P / N \equiv l_{N}$ independently of the particular vertices involved, where $P$ is the perimeter of the polygon.

We study the spin-dependent conductance of polygons by employing two different methods: A full-quantummechanical (FQM) approach, based on quantum graph theory ${ }^{5}$, and a much simpler description that accounts only for the spin dynamics at the lowest order in orbital winding by using the SRO of Eq. (2). The latter approach provides the spin-related AC phases accumulated by the carriers between input and output leads by following the two shortest possible paths (namely, the direct clockwise and counterclockwise paths neglecting any further winding and backscattering contributions). The corresponding quantum amplitude for transmission is calculated as the sum of successive application of the operator (2) along both paths, each of them composed by $N / 21 \mathrm{D}$ conducting bonds. This reads

$$
\begin{aligned}
\Gamma_{N}^{0}= & \mathcal{R}_{N / 2+1, N / 2} \ldots \mathcal{R}_{3,2} \cdot \mathcal{R}_{2,1}+ \\
& \mathcal{R}_{N / 2+1, N / 2+2} \ldots \mathcal{R}_{N-1, N} \cdot \mathcal{R}_{N, 1},
\end{aligned}
$$

where $\Gamma_{N}^{0}$ is a $2 \times 2$ matrix containing the spin-resolved amplitudes and the index 0 stands for lowest-ordercontribution. The first and second terms in Eq. (3) concern the clockwise and counterclockwise paths, respectively. The associated transmission coefficient (proportional to the linear conductance) is given by

$$
T_{N}^{0}=\operatorname{Tr}\left[\Gamma_{N}^{0} \Gamma_{N}^{0 \dagger}\right]
$$

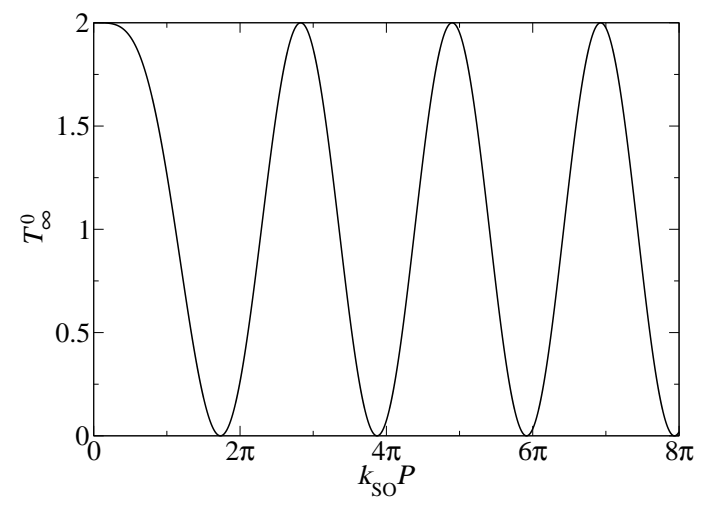

FIG. 3: Lowest order transmission as function of the dimensionless SO coupling strength for a polygon in the limit $N \rightarrow \infty$, equivalent to a circular ring.

where the trace runs over the spin degree of freedom. In Fig. 2 we plot $T_{N}^{0}$ as a function of the dimensionless SO strength $k_{\mathrm{SO}} P=\pi P / L_{\mathrm{SO}}$ for $N=4,6$ and 8 . We find a series of zeros showing up due to destructive quantum interference. This stems from the fact that spins following different paths acquire different $\mathrm{AC}$ phases according to the traveling direction ${ }^{3.4 .5}$. The curves present a periodicity equal to $N \pi$, proportional to the number of vertices. The number of zeros within a period equals $N / 2-1$. For $N>4$ it becomes evident the presence of two very different frequencies participating in the oscillatory pattern. The first one, associated to the shorter bond-length-scale $l_{N}$, determines the absolute period of the curves increasing linearly with $N$ as pointed out above. The second frequency is much higher and weakly dependent on $N$. This is related to the longer perimeter-length-scale $P$, giving rise to oscillations of period between $2 \pi$ and $3 \pi$. As a function of $N$, it approaches $2 \pi$ as $N$ increases.

These features are better understood by taking the limit $N \rightarrow \infty$, where the series of regular polygons converges to a circle (Fig. 1). Results for $T_{N}^{0}$ in the limit of large $N\left(T_{\infty}^{0}\right)$ are shown in Fig. [3] where we recover the results for a ring recently reported by Frustaglia and Richter in Ref. 4. There, we find that the series of zeros in Fig. 3 are placed at $\frac{17}{}$

$$
k_{\mathrm{SO}} P=\pi \sqrt{(2 n)^{2}-1},
$$

with $n$ integer. In contrast to the case of polygons, only higher frequency oscillations associated to the perimeter length scale show up. These appear to be quasi-periodic, with a period approaching $2 \pi$ as $k_{\mathrm{SO}} P \rightarrow \infty$. The latter corresponds to the adiabatic limit, where spins follow the local direction of the effective in-plane magnetic field during transport, and Berry phases arise $\mathrm{u}^{\underline{4}}$. Oscillations of period $2 \pi$ can consequently be identified with the adiabatic limit. From our discussion regarding results for finite $N$ in Fig. 2 (see above) we conclude that the limit of adiabatic spin transport is never really achieved in polygons, and it may be only approached for large $N$. This is due to the strongly non-adiabatic scattering that the 


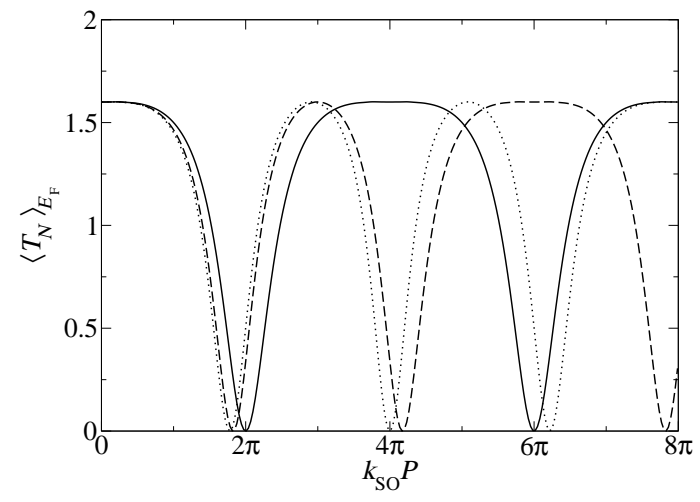

FIG. 4: Full average transmission as function of the dimensionless SO coupling strength for polygons with $N=4$ (solid line), $N=6$ (dashed line) and $N=8$ (dotted line). To be compared with the lowest order results of Fig. 2

spins suffer at the vertices as a consequence of the abrupt change of direction of the effective magnetic field. That is particularly relevant in square loops $(N=4)$ where the period of the oscillations doubles that for rings (compare Fig. 2 (solid line) with Fig. 31). This indicates that the rate of $\mathrm{AC}$ phase accumulation as a function of $k_{\mathrm{SO}} P$ is smaller for the square loop.

We finally present results using a FQM approach which takes into account higher order winding contributions and backscattering effects due to finite coupling to the leads ${ }^{5}$. Results are shown in Fig. 4 for polygons with $N=4,6$ and 8 and are to be compared with those of Fig. [2] for the SRO approach. There we plot the corresponding average transmission $\left\langle T_{N}\right\rangle_{E_{\mathrm{F}}}$. The average is performed on the Fermi energy $E_{\mathrm{F}}$ for a energy window larger than the mean level spacing. This is done in order to avoid energy-dependent features related to the presence of quasibound states which are formed in the polygons when the coupling to the leads is finite (see
Ref. 4 for a similar procedure). By comparing Fig. 4 with Fig. 2 we note that both approaches provide the same set of points of zero conductance. The main differences are in the shape and amplitude of the curves. This is due to the fact that in the simpler SRO approach we assumed an ideal coupling to the leads, and we consider only the lowest order terms in orbital winding. Higher order contributions, due to paths that go several times around the polygons, modify slightly the shape of the curves. Regarding the amplitude reduction, this is mainly due to backscattering at the incoming contact.

In conclusion, we studied the spin-dependent transport properties of $1 \mathrm{D}$ polygons subject to SO coupling. We showed that the polygons vertices act as scattering centers for spin, leading to highly non-adiabatic spin evolution. This hinders the spins to reach the limit of adiabatic spin transport where Berry phases manifest. For polygons with large number of vertices the adiabatic regime is restored for large coupling strength, in agreement with previous results for circular rings. It is interesting to note that by simply changing the shape of the loop from a square to a circle one can double the frequency of the oscillation pattern as a function of the dimensionless SO coupling strength. Moreover, we remark that a simple approach based on SRO captures the relevant spin dynamics, giving the exact positions of the conductance zeros. We finally note that our results are not expected to suffer any significative change in the presence of residual disorder, as far as the elastic mean free path stays of the order of the semi-perimeter $P / 2$ (i.e., no new length scales are introduced). Some effects would evetually manifest on the amplitude of the oscillations, where the minima would not reach zero due to the symmetry breaking introduced by disorder.

Acknowledgments: This work was partly supported by the EU Spintronics Research Training Network (DF).
1 S. Datta and B. Das, Appl. Phys. Lett. 56, 665 (1990).

2 E. Rashba, Fiz. Tverd. Tela (Leningrad) 2, 1224 (1960), [Sov. Phys. Solid State 2, 1109 (1960)].

3 H. Mathur and A.D. Stone, Phys. Rev. Lett. 68, 2964 (1992); Y. Oreg and O. Entin-Wohlman, Phys. Rev. B 46, 2393 (1992); J. Nitta, F.E. Meijer, and H. Takayanagi, Appl. Phys. Lett. 75, 695 (1999); S. Souma and B.K. Nikolić, Phys. Rev. B 70, 195346 (2004).

4 D. Frustaglia and K. Richter, Phys. Rev. B 69, 235310 (2004).

5 D. Bercioux, M. Governale, V. Cataudella, V. Marigliano Ramaglia Phys. Rev. Lett. 93, 56802 (2004).

6 Y. Aharonov and A. Casher, Phys. Rev. Lett. 53, 319 (1984).

7 I. Žutić, J. Fabian, and S. Das Sarma, Rev. Mod. Phys. 76, 323 (2004).

8 D. Loss, P. Goldbart, and A.V. Balatsky, Phys. Rev. Lett. 65, 1655 (1990); A. Stern, Phys. Rev. Lett. 68, 1022
(1992); A.G. Aronov and Y.B. Lyanda-Geller, Phys. Rev. Lett. 70, 343 (1993); A.G. Mal'shukov, V.V. Shlyapin, and K.A. Chao, Phys. Rev. B 60, R2161 (1999); D. Frustaglia and K. Richter, Found. Phys. 31, 399 (2001).

9 M.V. Berry, Proc. R. Soc. London 392, 45 (1984).

10 A.F. Morpurgo, J.P. Heida, T.M. Klapwijk, B.J. van Wees, and G. Borghs, Phys. Rev. Lett. 80, 1050 (1998); J.-B. Yau, E.P. De Poortere, and M. Shayegan, Phys. Rev. Lett. 88, 146801 (2002).

11 M.J. Yang, C.H. Yang, and Y.B. Lyanda-Geller, Europhys. Lett. 66, 826 (2004).

12 For an updated discussion see M. Popp, D. Frustaglia, and K. Richter, Phys. Rev. B 68, 041303(R) (2003).

13 M.J. van Veenhuizen, T. Koga, and J. Nitta, cond-mat/0412609

14 R. Landauer, IBM J. Res. Dev. 1, 223 (1957); M. Buttiker, IBM J. Res. Dev. 32, 317 (1988).

15 F. Mireles and G. Kirczenow, Phys. Rev. B 64, 24426 
(2001); M. Governale and U. Zülicke, Phys. Rev. B 66, 073311 (2002).

16 J. Nitta, T. Akazaki, H. Takayanagi, and T. Enoki, Phys. Rev. Lett. 78, 1335 (1997); T. Schäpers, J. Engels, T. Klocke, M. Hollfelder, and H. Lüth, J. Appl. Phys. 83,
4324 (1998); D. Grundler, Phys. Rev. Lett. 84, 6074 (2000).

17 Note that the adiabaticity parameter $Q_{\mathrm{R}}$ defined in Ref. 4 corresponds to our $k_{\mathrm{SO}} P / \pi$. 